OPEN ACCESS

Edited by:

Laurent Rénia,

Technology and Research (A*STAR), Singapore

Reviewed by:

Antonella Folgori,

Okairos AG, Italy

Christopher M. Jewell,

University of Maryland,

College Park, United States

${ }^{*}$ Correspondence:

Xiubao Ren

renxiubao@tjmuch.com

Specialty section:

This article was submitted

to Vaccines and Molecular

Therapeutics,

a section of the journal

Frontiers in Immunology

Received: 17 July 2017 Accepted: 06 February 2018

Published: 07 March 2018

Citation:

Zhao H, XU C, Luo X, Wei F, Wang N,

Shi $H$ and Ren X (2018)

Seroprevalence of Neutralizing

Antibodies against Human

Adenovirus Type- 5 and

Chimpanzee Adenovirus Type-68

in Cancer Patients.

Front. Immunol. 9:335.

doi: 10.3389/fimmu.2018.00335

\section{Seroprevalence of Neutralizing Antibodies against Human Adenovirus Type-5 and Chimpanzee Adenovirus Type-68 in Cancer Patients}

\author{
Hua Zhao ${ }^{1,2,3,4,5}$, Can $X^{6,7}$, Xiaoli Luo ${ }^{6,7}$, Feng Wei ${ }^{1,2,3,4,5}$, Ning Wang $^{6,7}$, Huiying Shi ${ }^{6,7}$ \\ and Xiubao Ren ${ }^{1,2,3,4,5 *}$ \\ ${ }^{1}$ Department of Immunology, Tianjin Medical University Cancer Institute \& Hospital, Tianjin, China, ${ }^{2}$ National Clinical \\ Research Center for Cancer, Tianjin, China, ${ }^{3}$ Key Laboratory of Cancer Prevention and Therapy, Tianjin, China, \\ ${ }^{4}$ Tianjin's Clinical Research Center for Cancer, Tianjin, China, ${ }^{5}$ Key Laboratory of Cancer Immunology and Biotherapy, \\ Tianjin, China, ${ }^{6}$ Bioroc Pharmaceutical \& Biotech Company, Tianjin, China, ${ }^{7}$ Tianjin Genstar Vaccine Limited Liability \\ Company, Tianjin, China
}

Since the preclinical results about chimpanzee adenovirus serotype-68 (AdC68)-based vaccine showed an encouraging results, it reminded us that AdC68 may be a suitable cancer vaccine vector. Previous study indicated that the seroprevalence of neutralizing antibodies (NAbs) against adenovirus was different between cancer patients and healthy volunteers. Knowledge regarding the prevalence rates of AdC68 NAbs for cancer patients is lacking. Therefore, assessing the preexistence of NAbs against AdC68 in cancer patients could provide useful insights for developing future AdC68-based cancer vaccines. In this study, 440 patients with different pathological types of tumors and 204 healthy adult volunteers were enrolled to evaluate the NAbs against AdC68 and human adenovirus serotype-5 (AdHu5). The seroprevalence of NAbs against AdC68 was much lower than that against AdHu5 in cancer subjects (43.64 vs. $67.05 \%, P<0.01$ ). The seroprevalence rates of NAbs to AdC68 in the cancer subjects were statistically higher than those detected in the healthy adult volunteers (43.64 vs. $23.53 \%, P=0.000)$. The seroprevalence rates of AdC68 NAbs were much lower in lung, laryngeal, esophageal, and cervical cancer patients compared with oropharyngeal, colon, and rectal cancer patients. Furthermore, the seroprevalence rates of AdC68 NAbs were much lower in lung adenocarcinoma patients than in lung squamous cell carcinoma patients (35.00 vs. $70.00 \%, P<0.05)$. No significant difference in the AdC68 NAbs among patients with different clinical stages of cancer was detected. The percentage of NAbs against AdC68 was significantly lower than that against AdHu5 $(P<0.05)$ in stage-I, -II, and -III cancer patients. No significant difference between the percentage of NAbs against AdC68 and AdHu5 in the subjects with stage-IV cancer was detected. The study also demonstrated the distribution of AdHu5 and AdC68 NAb titers for the positive samples. It showed that very low NAb titers against AdC68 with respect to AdHu5 in both healthy subjects and cancer subjects, especially in lung, laryngeal, esophageal, gastric, and 
cervical carcinomas. Also, the titer of NAbs against AdC68 was significantly lower than that against AdHu5 in the same clinical stage and age group $(P<0.05)$. Taken together, the present study showed that NAbs against AdC68 is much lower than AdHu5, especially in lung adenocarcinoma, laryngeal cancer, esophageal cancer, and cervical cancer patients. These results provided strong support for candidating AdC68 as a suitable vector of cancer vaccines.

Keywords: chimpanzee adenovirus serotype-68, human adenovirus serotype-5, neutralizing antibodies, cancer vaccine, cancer immunotherapy

\section{INTRODUCTION}

Therapeutic cancer vaccines based on replication-defective adenoviral vectors have been developing with varying levels of success. Functional tumor antigen-specific $\mathrm{CD}^{+} \mathrm{T}$ cells should be activated to achieve tumor regression $(1,2)$. Adenoviral vectors derived from the common human adenovirus serotype- 5 (AdHu5) vector are considered the most well-studied vector because of their ability to induce potent transgene productspecific T- and B-cell responses in experimental animals (3-5) and clinical trials as carriers for HIV-1 vaccines (6). However, the neutralizing antibodies (NAbs) to AdHu5 virus are common in humans and therefore the immune response of the transgene products is often dampened (7-9). To circumvent the problem of preexisting immunity, nonhuman adenovirus vectors, which isolated from chimpanzees with NAbs of lower seroprevalence than that of AdHu5, have been well characterized and developed as vaccine carriers (10-13).

The chimpanzee adenovirus serotype-68 (AdC68) has been shown to be a good gene carrier in vaccine development owing to its high transduction efficiency, broad cell tropism, high gene expression, good genetic stability, and low seropositive rate in humans $(13,14)$. It can grow in HEK293 cells as sufficient AS and exhibits no significant cross-neutralization by sera against human serotypes including AdHu2, 4, 5, 7, and 12 (10). Like AdHu5, previous study demonstrated thatAdC7- and AdC68based vaccines could also induce strong $\mathrm{T}$ - and $\mathrm{B}$-cell responses to exogenous antigens in mice and nonhuman primates (15). Various vaccine candidates based on AdC68 have been developed for controlling different infectious diseases, including influenza of H5N1 and H7N9 $(16,17)$. Preclinical study has also demonstrated that the hexon-modified AdC68-based vaccine can induce immune protection against EV71 and CA16 challenge in mice (18). An AdC68-based rabies virus vaccine, termed AdC68rab.gp, induced sustained central and mucosal antibody responses to rabies virus and provides complete protection against rabies virus challenge (19). Early study also reported that AdHu5 vector-based vaccine induced a potent transgene product-specific CD $8+$ T-cell response that can be increased substantially by using AdC68 vector-based vaccine expressing the same transgene product for booster immunization. This type of heterologous prime-boost regimen is far more effective than priming with a DNA vaccine followed by booster immunization with an Ad recombinant or by using the same Ad vaccine carrier repeatedly (20). The AdC68-based vaccine could also induce protective effector and memory T-cell responses against malignant melanoma cells in mice (21).

As we know, there were no clinical trials based on AdC68 vectors. Since the preclinical results about AdC68-based vaccine showed an encouraging result, it reminded us that AdC68 may be a suitable cancer vaccine vector. However, previous study indicated that the seroprevalence of NAbs against adenovirus was different between cancer patients and healthy volunteers (22). The studies comparing the prevalence of NAbs specific for AdHu 5 and AdC68 have mainly been limited to healthy human subjects $(13,20)$. Knowledge regarding the prevalence rates of AdC68 NAbs for cancer patients is lacking. Assessing the preexistence of NAbs against AdC68 in cancer patients could provide useful insights for developing future AdC68-based cancer vaccines.

\section{MATERIALS AND METHODS}

\section{Human Samples}

Human serum and plasma samples were obtained from 204 healthy adult volunteers and 440 cancer patients from Tianjin Cancer Hospital, including eight types of cancer patients: 80 lung cancer patients, 35 laryngeal cancer patients, 9 oropharyngeal cancer patients, 76 esophageal cancer patients, 40 gastric cancer patients, 120 cervical cancer patients, 40 colon cancer patients, and 40 rectal cancer patients. All patients enrolled in this study were pathologically diagnosed according to the National Comprehensive Cancer Network Clinical Practice Guidelines. All serum samples were stored at $-80^{\circ} \mathrm{C}$ prior to use, and sera/plasma were heat inactivated at $56^{\circ} \mathrm{C}$ for $30 \mathrm{~min}$.

\section{Recombinant Adenoviruses}

Replication-defective, green fluorescent protein (GFP)-expressing adenovirus vectors derived from serotypes AdHu5 and AdC68 were purchased from Jikai (Shanghai, China) and Bioroc (Tianjin, China), respectively.

\section{Adenovirus Neutralization Assay}

Adenovirus neutralization assays were performed as described previously (20). Briefly, heat-inactivated sera were added to 96-well plates after serial doubling dilutions $(1: 10-1: 1,280)$ were mixed with $1 \times 10^{7}$ AdHu5 and AdC68 virus particles expressing GFP and incubated for $1 \mathrm{~h}$ at $37^{\circ} \mathrm{C}$ in a $5 \% \mathrm{CO}_{2}$ atmosphere. 
Dulbecco minimum essential medium (DMEM) was used as the negative control. Serum from $B A L B / c$ mice primed and boosted via the intramuscular route with $1 \times 10^{11} \mathrm{GFP}$-expressing AdHu5 and AdC68 viral particles were used as positive controls. In total, $2.5 \times 10^{4}$ HEK293 cells per well were added to the 96-well plate and mixed fully with the medium. The results were read after $24 \mathrm{~h}$ of incubation. The NAb titers are expressed as the reciprocal of dilution in which the ratio of GFP-expressing cells was reduced to approximately $50 \%$ compared with that of the negative control. Titers $>20$ were scored as positive for the presence of serotypespecific NAbs.

\section{Statistical Analysis}

Statistical Package for the Social Sciences version 21.0 (IBM Corp., USA) was applied to perform all the statistical analysis. The chi-square test was used to compare the seroprevalence rates of AdHu5 and AdC68. The Friedman ANOVA test was used to compare the NAb titers among the adenoviral serotypes. In all the tests, the values of $P<0.05$ using two-sided tests were considered statistically significant.

\section{RESULTS}

\section{Study Participants and Characteristics}

The characteristics of the cancer patient and healthy adult volunteer participants are detailed in Table 1. Sex and age were comparable between the cancer patients and healthy adult volunteers.

\section{Seroprevalence of Anti-Adenovirus NAbs}

In this study, 204 samples from healthy adult volunteers and 440 samples from cancer patients were evaluated for their NAbs against AdHu5 and AdC68. The seroprevalence rates in the total samples of NAbs against AdHu5 were statistically higher than that of AdC68 (68.48 vs. $37.27 \%, P<0.01$, Table 2 ). In the group of healthy adult volunteers, the seroprevalence rates of NAbs against AdHu5 were statistically higher than that of AdC68 ( 71.57 vs. $23.53 \%, P<0.01$ ). In the group of cancer patients, the seroprevalence rates of NAbs against AdHu5 were statistically higher than that of AdC68 (67.05 vs. 43.64\%, $P<0.01$ ). Taken

TABLE 1 | Demographics of study participants with assayed samples $(N=644)$.

\begin{tabular}{cc} 
Cancer patients & Healthy adult volunteers \\
\hline$(n, \%)$ & $(n, \%)$
\end{tabular}

\begin{tabular}{lc}
\hline Age groups (years) & \\
$<60$ & $258(58.64 \%)$ \\
$\geq 60$ & $182(41.36 \%)$ \\
Gender & \\
Male & $228(51.82 \%)$ \\
Female & $212(48.18 \%)$ \\
Pathological type & \\
Squamous cell carcinoma & $280(63.64 \%)$ \\
Adenocarcinoma & $160(36.36 \%)$ \\
Clinical stage & \\
Stage I & $133(30.23 \%)$ \\
Stage II & $162(36.82 \%)$ \\
Stage III & $115(26.14 \%)$ \\
Stage IV & $30(6.82 \%)$
\end{tabular}

together, these results indicate that preexisting immunity is more common against AdHu5 than against AdC68, suggesting the use of AdC68 vectors rather than AdHu5 vectors as vaccine carriers.

No significant difference in the seroprevalence rates of NAbs against AdHu 5 between healthy adult volunteers and cancer patients existed $(P>0.05)$. However, the seroprevalence rates of NAbs against AdC68 in the cancer patient samples were statistically higher than that of healthy adult volunteers ( 43.64 vs. $23.53 \%, P=0.000$ ). The results indicated that preexisting immunity against AdC68 is more common in cancer patients than in healthy volunteers.

In this study, we further analyzed the distribution of AdHu5 and AdC68 NAb titers for the positive samples (Figure 1, detailed data showed in Table S1 in Supplementary Material). The NAb titers were stratified across the following tiers: low titers $(\geq 20$ and $\leq 160)$, medium titers $(\geq 320$ and $\leq 640)$, and high titers $(\geq 1,280)$. For all of the human subjects, the AdHu5 NAb titers for positive samples from low to high titers were 51.02, 33.79, and $15.19 \%$, respectively. In contrast, the AdC68 NAb titers for positive samples from low to high titers were 96.67, 3.33, and $0 \%$, respectively. For the healthy adult volunteers, the AdHu5 $\mathrm{NAb}$ titers for positive samples from low to high titers were $32.19,35.62$, and $32.19 \%$, respectively, whereas all of the positive AdC68 NAb sample titers were lower or equal to 160. Most cancer patient subjects exhibited low AdHu5 NAb titers ( $\geq 20$ and $\leq 160$, $60.34 \%$ ), and a small fraction exhibited very high AdHu 5 NAb titers $(\geq 1,280,6.78 \%)$. In contrast to this distribution, most cancer patient subjects exhibited low NAb titers against AdC68 $(\geq 20$ and $\leq 60,95.83 \%$ ), and a small fraction of cancer patients exhibit a medium level of AdC68 NAb titers ( $\geq 320$ and $\leq 640,4.17 \%$ ).

\section{Seroprevalence of Anti-Adenovirus NAbs in Different Cancer Types}

Subjects with eight different types of cancer were evaluated for their levels of AdHu5 and AdC68 NAbs (Table 3). The percentages of NAbs against AdC68 were significantly lower in lung, laryngeal, esophageal, and cervical carcinoma patients than those against AdHu5 $(P<0.05)$. No significant differences between the percentages of NAbs against AdC68 and AdHu5 in oropharyngeal, gastric, rectal, or colon cancer patients were detected. These results indicate that preexisting immunity against AdC68 is rare in laryngeal, esophageal, and cervical carcinomas, which suggests that AdC68 vectors may be considered vaccine carriers in these types of cancers.

We also analyzed the distribution of AdHu5 and AdC68 NAb titers for positive samples in the different types of cancer subjects (Table 4). For the subjects with lung, laryngeal, esophageal, gastric, and cervical carcinomas, the distributions of AdHu $5 \mathrm{NAb}$ titers for positive samples were different from the distribution of AdC68 NAb titers $(P<0.05)$. No differences in the distributions between the AdHu5 and AdC68 NAb titers for positive subjects with oropharyngeal, rectal, or colon cancer were detected.

TABLE 2 | Seroprevalence of AdHu5 and AdC68 neutralizing antibody in cancer patients and healthy adult volunteers.

\begin{tabular}{llll}
\hline & \multicolumn{1}{c}{ AdHu5 } & \multicolumn{1}{c}{ AdC68 } & P-value \\
\hline Total samples & $68.48 \%(441 / 644)$ & $37.27 \%(240 / 644)$ & 0.000 \\
Healthy adult Volunteers & $71.57 \%(146 / 204)$ & $23.53 \%(48 / 204)$ & 0.000 \\
Cancer patients & $67.05 \%(295 / 440)$ & $43.64 \%(192 / 440)$ & 0.000
\end{tabular}




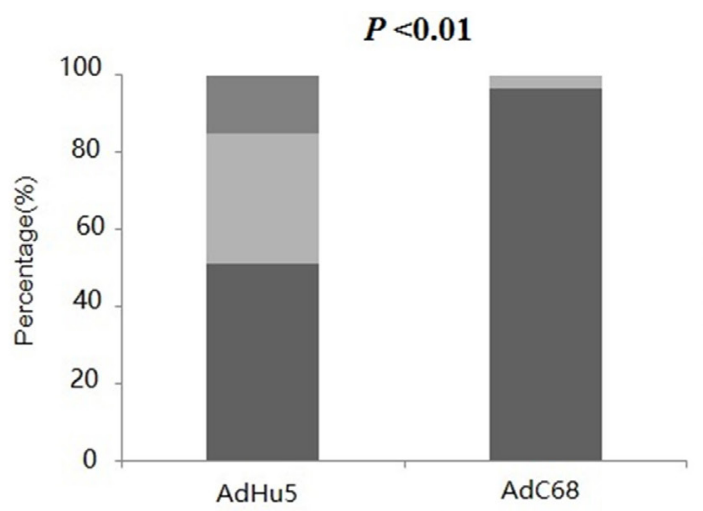

Total Human Samples

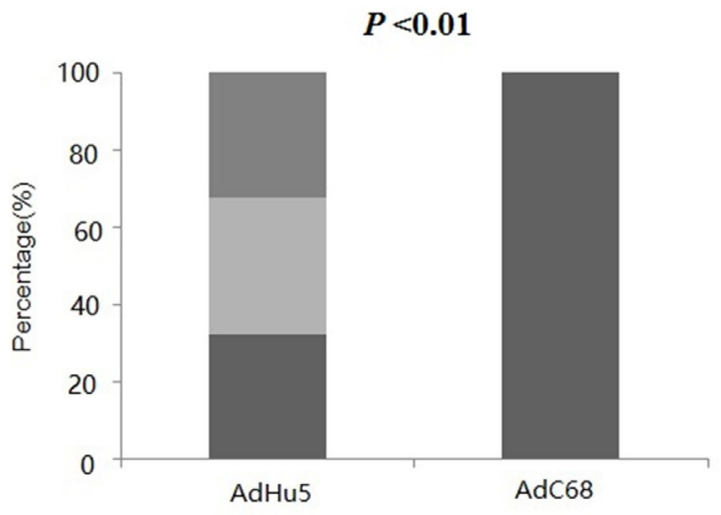

Healthy Human Samples

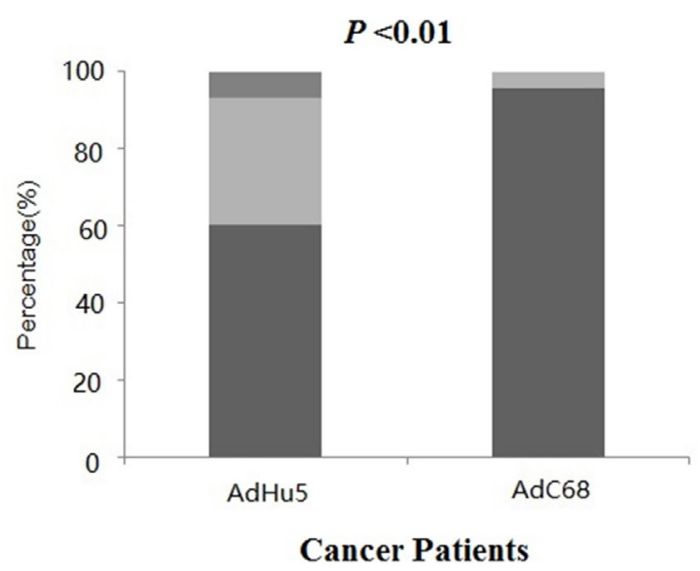

Neutralizing antibody titer

- $\geqslant 1280$

= $320-640$

- 20-160

FIGURE 1 | Distribution of neutralizing antibody (NAb) titers against AdHu5 and AdC68 in positive samples. NAb titers were stratified in the following tiers: low titer ( $\geq 20$ and $\leq 160)$, medium titer $(\geq 320$ and $\leq 640)$, and high titer $(\geq 1,280)$. Across positive samples from all of the human subjects, the NAb titers against AdHu5 from low to high were 51.02, 33.79, and 15.19\%, respectively. The AdC68 NAb titers in positive samples from low to high were 96.67, 3.33, and 0\%, respectively, which was significantly different from AdHu5 $(P<0.01)$. For positive samples from the cancer subjects, the NAb titers against AdHu5 from low to high were $51.02,33.79$, and $15.19 \%$, respectively. However, most cancer subjects exhibited low NAb titers against AdC68 ( $\geq 20$ and $\leq 160,95.83 \%)$, and a small fraction of cancer subjects exhibited a medium level of AdC68 NAb titers ( $\geq 320$ and $\leq 640,4.17 \%$ ). For the healthy adult volunteers, the AdHu5 NAb titers in the positive samples from low to high were $32.19,35.62$, and 32.19\%, respectively, whereas all of titers from the positive NAb samples against AdC68 were lower or equal to 160.

TABLE 3 | Seroprevalence of AdHu5 and AdC68 neutralizing antibody in patients with different types of cancer.

\begin{tabular}{llll}
\hline & \multicolumn{1}{c}{ AdHu5 } & \multicolumn{1}{c}{ AdC68 } & P-value \\
\hline Lung cancer & $75.00 \%(60 / 80)$ & $52.50 \%(42 / 80)$ & 0.003 \\
Laryngeal cancer & $88.57 \%(31 / 35)$ & $25.71 \%(9 / 35)$ & 0.000 \\
Oropharyngeal cancer & $66.67 \%(6 / 9)$ & $66.67 \%(6 / 9)$ & 1.000 \\
Esophageal cancer & $67.11 \%(51 / 76)$ & $32.89 \%(25 / 76)$ & 0.000 \\
Gastric cancer & $77.50 \%(31 / 40)$ & $75.00 \%(30 / 40)$ & 0.793 \\
Cervical carcinoma & $54.17 \%(65 / 120)$ & $26.67 \%(32 / 120)$ & 0.000 \\
Rectal cancer & $65.00 \%(26 / 40)$ & $70.00 \%(28 / 40)$ & 0.633 \\
Colon cancer & $62.50 \%(25 / 40)$ & $50.00 \%(20 / 40)$ & 0.260 \\
\hline
\end{tabular}

\section{Seroprevalence of Anti-Adenovirus NAbs in Cancer Patients of Different Clinical Stages}

We further analyzed the seroprevalence of AdHu5 and AdC68 NAbs in cancer patients in different clinical stages of their diseases (Table 5). No significant difference in the AdC68 NAbs was detected among patients of different disease stages $(P=0.849)$. The percentages of NAbs against AdC68 were significantly lower than those against AdHu5 $(P<0.05)$ in subjects of stages I, II, and III. No significant difference in the percentages of NAbs against AdC68 and AdHu5 in subjects of stage IV was detected.

We also analyzed the distribution of AdHu5 and AdC68 titers for positive samples in different clinical stages, and a significant difference between these two vectors was detected $(P<0.05$, Figure 2, detailed data showed in Table S1 in Supplementary Material). For stage-I positive subjects, the AdHu5 NAb titers from low to high were $58.54,34.15$, and $7.32 \%$, respectively, while the AdC68 NAb titers from low to high were 94.55, 5.45, and $0 \%$, respectively. A significant difference between AdHu5 NAb and AdC68 Nab titers was detected in stage-I positive subjects $(P<0.01)$. For stage-II positive subjects, the AdHu 5 NAb titers from low to high were $64.81,29.63$, and 5.56\%, respectively, while all of the positive AdC68 NAb sample titers were lower than or equal to 160, which was significantly different from AdHu5 $(P<0.01)$. For stage-III positive subjects, the AdHu5 NAb titers 
TABLE 4 | Distribution of AdHu5 and AdC68 neutralizing antibody titers in positive samples from subjects from different type of cancer.

\begin{tabular}{|c|c|c|c|c|}
\hline & High (20-160 titers) & Medium (320-640 titers) & Low $(\geq 1,280$ titers $)$ & $P$-value \\
\hline Lung cancer & & & & 0.000 \\
\hline AdHu5 neutralizing antibody & 37 (61.67\%) & 18 (30.00\%) & $5(8.33 \%)$ & \\
\hline AdC68 neutralizing antibody & 40 (95.24\%) & $2(4.76 \%)$ & 0 & \\
\hline Laryngeal cancer & & & & 0.005 \\
\hline AdHu5 neutralizing antibody & $14(45.16 \%)$ & 17 (54.84\%) & 0 & \\
\hline AdC68 neutralizing antibody & 9 (100.0\%) & 0 & 0 & \\
\hline Oropharyngeal cancer & & & & 0.301 \\
\hline AdHu5 neutralizing antibody & $4(66.66 \%)$ & $1(16.67 \%)$ & $1(16.67 \%)$ & \\
\hline AdC68 neutralizing antibody & $6(100.0 \%)$ & 0 & 0 & \\
\hline Esophageal cancer & & & & 0.028 \\
\hline AdHu5 neutralizing antibody & $22(42.31 \%)$ & $23(44.23 \%)$ & $7(13.46 \%)$ & \\
\hline AdC68 neutralizing antibody & $6(100.0 \%)$ & 0 & 0 & \\
\hline Gastric cancer & & & & 0.011 \\
\hline AdHu5 neutralizing antibody & 25 (80.65\%) & $6(19.35 \%)$ & 0 & \\
\hline AdC68 neutralizing antibody & $30(100.0 \%)$ & 0 & 0 & \\
\hline Cervical carcinoma & & & & 0.001 \\
\hline AdHu5 neutralizing antibody & 39 (60.0\%) & $20(30.77 \%)$ & $6(9.23 \%)$ & \\
\hline AdC68 neutralizing antibody & $31(96.88 \%)$ & $1(3.12 \%)$ & 0 & \\
\hline Rectal cancer & & & & 0.496 \\
\hline AdHu5 neutralizing antibody & 20 (76.92\%) & $5(19.23 \%)$ & $1(3.85 \%)$ & \\
\hline AdC68 neutralizing antibody & $24(85.71 \%)$ & $4(14.29 \%)$ & 0 & \\
\hline Colon cancer & & & & 0.077 \\
\hline AdHu5 neutralizing antibody & 17 (68.0\%) & 7 (28.0\%) & $1(4.0 \%)$ & \\
\hline AdC68 neutralizing antibody & 19 (95.0\%) & $1(5.0 \%)$ & 0 & \\
\hline
\end{tabular}

TABLE 5 | Seroprevalence of AdHu5 and AdC68 neutralizing antibodies in subjects with different clinical stage of cancer.

\begin{tabular}{llll}
\hline Clinical stage & \multicolumn{1}{c}{ AdHu5 } & \multicolumn{1}{c}{ AdC68 } & P-value \\
\hline Stage I & $61.65 \%(82 / 133)$ & $41.35 \%(55 / 133)$ & 0.001 \\
Stage II & $66.67 \%(108 / 162)$ & $43.83 \%(71 / 162)$ & 0.000 \\
Stage III & $72.17 \%(83 / 115)$ & $44.35 \%(51 / 115)$ & 0.000 \\
Stage IV & $73.33 \%(22 / 30)$ & $50.00 \%(15 / 30)$ & 0.063 \\
\hline
\end{tabular}

from low to high were $61.45,32.53$, and $6.02 \%$, respectively. However, most cancer patient subjects exhibited low NAb titers against AdC68 ( $\geq 20$ and $\leq 160,94.12 \%)$ and a small fraction of cancer patients exhibited a medium level of AdC68 NAb titers ( $\geq 320$ and $\leq 640,5.88 \%$ ). The distribution of these two vectors in stage-III patients was significantly different $(P<0.01)$. For stageIV positive subjects, the AdHu5 NAb titers from low to high were 40.91, 45.45, and 13.64\%, respectively. However, the AdC68 NAb titers from low to high were $86.67,13.33$, and $0 \%$, respectively, which was significantly different than that of AdHu5 $(P<0.05)$.

\section{Seroprevalence of Anti-Adenovirus NAbs in Subjects with Different Pathological Types of Cancer}

In this study, we also analyzed the seroprevalence of anti-adenovirus NAbs in subjects with different pathological types of cancer. Because the laryngeal, oropharyngeal, esophageal, and cervical cancers were all squamous cell carcinomas, the seroprevalence rates of AdHu5 and AdC68 NAbs in squamous cell carcinomas could be shown together in Table 2. Because the gastric, rectal, and colon cancers were all adenocarcinomas, such as the squamous cell carcinomas, the seroprevalence of AdHu5 and AdC68 NAbs in adenocarcinomas could be the same within these three cancer subjects. Therefore, we analyzed the seroprevalence of $\mathrm{AdHu} 5$ and AdC68 NAbs in different pathological types of lung cancer.

The seroprevalence of AdHu5 NAbs in lung adenocarcinoma subjects was significantly higher than that of AdC68 (77.50 vs. $35.00 \%, P<0.01$ Table 6). In contrast, no difference between the seroprevalence of AdHu5 and AdC68 in the lung squamous cell carcinoma subjects was detected ( 72.50 vs. $70.00 \%, P>0.05)$. No significant difference in the seroprevalence rates of NAbs against AdHu5 between the lung adenocarcinoma and lung squamous cell carcinoma subjects was detected ( 77.50 vs. $72.50 \%, P=0.606$ ). However, the seroprevalence rates of NAbs against AdC68 in the samples from lung squamous cell carcinoma subjects were statistically higher than those from lung adenocarcinoma patients (70.00 vs. $35.00 \%, P=0.049$ ).

We further analyzed the distribution of AdHu5 and AdC68 $\mathrm{NAb}$ titers among the positive lung cancer subjects (Figure 3). For the positive lung squamous cell carcinoma samples, the AdHu5 $\mathrm{NAb}$ titers from low to high were 48.28, 37.93, and $13.79 \%$, respectively, while the AdC68 NAb titers from positive samples from low to high were $92.86,7.14$, and $0 \%$, respectively, which was significantly different from AdHu $5(P<0.05)$. For the positive lung adenocarcinoma samples, the AdHu $5 \mathrm{NAb}$ titers from low to high were $74.19,22.58$, and $3.23 \%$, respectively, while all of the positive AdC68 NAb sample titers were lower or equal to 160 . However, no significant difference between the distributions of the two vectors was detected in lung adenocarcinoma subjects $(P>0.05)$.

\section{Seroprevalence of Anti-Adenovirus NAbs in Different Age Groups}

In this study, we randomly collected peripheral plasma of 440 cancer patients so that the subjects were not evenly distributed according to the age groups. The subjects were divided into four 


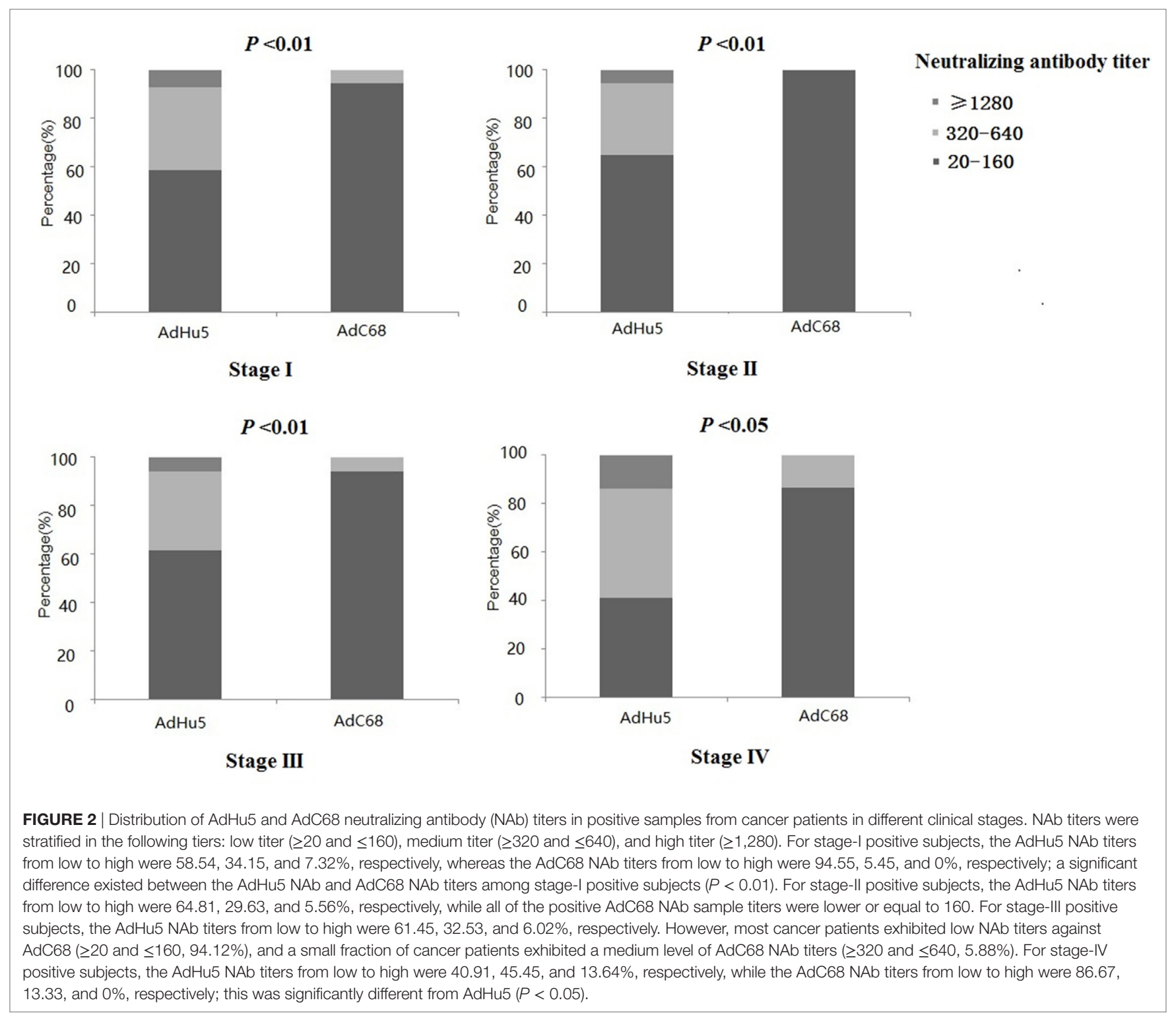

TABLE 6 | Seroprevalence of AdHu5 and AdC68 neutralizing antibody in subjects with different pathological type of lung cancer.

\begin{tabular}{lccc}
\hline & AdHu5 & AdC68 & P-value \\
\hline Squamous cell carcinoma & $72.50 \%(29 / 40)$ & $70.00 \%(28 / 40)$ & 0.805 \\
Adenocarcinoma & $77.50 \%(31 / 40)$ & $35.00 \%(14 / 40)$ & 0.008 \\
\hline
\end{tabular}

groups according to their age: 18-39 years of group, 40-49 years of group, 50-59 years of group, and no less than 60 years of group. The seroprevalence rates of NAbs against AdHu5 in the total samples and cancer samples were significantly increased with the increasing age ( $P=0.038$ and 0.005 , respectively), so as to the AdC68 ( $P=0.031$ and 0.006 , respectively). However, the seroprevalence rates of NAbs against AdHu5 and AdC68 showed no significant difference in the healthy adult volunteers $(P>0.05)$. The seroprevalence rates of NAbs against AdC68 were much higher than AdHu5 in the same age groups except for cancer patients of $18-39$ years of group $(P<0.05$, Table 7$)$. Furthermore, there were no significant differences in the distribution of $\mathrm{AdHu} 5$ and AdC68 NAb titers for positive samples in the different age groups of neither the total subjects, healthy subjects, nor cancer subjects $(P>0.05$, Table 7$)$.

In addition, the titer of NAbs against AdC68 was significantly lower than that against AdHu 5 in the same age groups $(P<0.05$, Table 8). However, there were no significant differences of the titer of NAbs against AdHu5 and AdC68 in different age groups $(P>0.05$, Table 8).

\section{DISCUSSION}

Prior studies reported that AdC68 NAbs occur at a lower prevalence in healthy volunteers from North America (2-4\%), Southeast Asia (1.5-3\%), Africa (2-20\%), and China (9.4-21.8\%) (23-25), which are much lower than the NAbs to AdHu5 


\section{$P<0.01$}

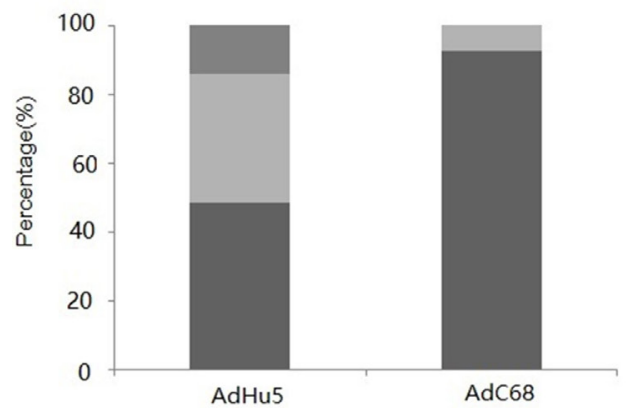

Lung Squamous Cell Carcinoma
$P>0.05$

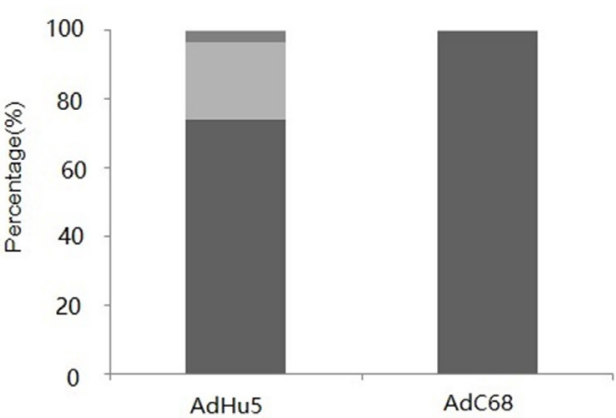

Lung Adenocarcinoma
Neutralizing antibody titer

- $\geqslant 1280$

" 320-640

- $20-160$

FIGURE 3 | Distribution of AdHu5 and AdC68 neutralizing antibody (NAb) titers in positive samples from lung squamous cell carcinoma and adenocarcinoma subjects. NAb titers were stratified in the following tiers: low titer ( $\geq 20$ and $\leq 160)$, medium titer $(\geq 320$ and $\leq 640)$, and high titer ( $\geq 1,280)$. For the positive samples from lung squamous cell carcinoma patients, the AdHu5 NAb titers from low to high were 48.28, 37.93, and 13.79\%, respectively, while the AdC68 NAb titers from low to high were $92.86,7.14$, and $0 \%$, respectively; this was significantly different from AdHu $(P<0.05)$. For positive samples from lung adenocarcinoma patients, the AdHu5 NAb titers from low to high were 74.19, 22.58, and 3.23\%, respectively, while all of the positive AdC68 NAb sample titers were lower or equal to 160. However, no significant difference in the distribution of the two vectors in lung adenocarcinoma patients was observed $(P>0.05)$.

TABLE 7 | Seroprevalence of AdHu5 and AdC68 neutralizing antibody in different age groups.

\begin{tabular}{lrrrr}
\hline Age groups (years) & $\boldsymbol{N}$ & AdHu5 (\%) & AdC68 (\%) & $P$-value \\
\hline $\begin{array}{l}\text { Total samples } \\
\text { 18-39 }\end{array}$ & 36 & $20(55.56)$ & $9(25.00)$ & 0.008 \\
$40-49$ & 113 & $69(61.06)$ & $32(28.32)$ & 0.000 \\
50-59 & 218 & $152(69.72)$ & $84(38.53)$ & 0.000 \\
$\geq 60$ & 274 & $200(72.99)$ & $115(41.97)$ & 0.000 \\
Healthy adult volunteers & & & & \\
18-39 & 11 & $8(72.73)$ & $1(9.09)$ & 0.002 \\
40-49 & 27 & $18(66.67)$ & $7(25.93)$ & 0.003 \\
50-59 & 74 & $58(78.38)$ & $16(21.62)$ & 0.000 \\
$\geq 60$ & 92 & $62(67.39)$ & $24(26.09)$ & 0.000 \\
Cancer patients & & & & \\
18-39 & 25 & $12(48.00)$ & $8(32.00)$ & 0.248 \\
$40-49$ & 86 & $51(59.30)$ & $25(29.07)$ & 0.000 \\
50-59 & 144 & $94(65.28)$ & $68(47.22)$ & 0.002 \\
$\geq 60$ & 182 & $138(75.82)$ & $91(50.00)$ & 0.000 \\
\hline
\end{tabular}

seroprevalence rates $(60-100 \%)$ in the same regions $(26-30)$. In the present study, AdHu5 NAb prevalence of $68.48 \%$ among all the human subjects $(n=644)$ was similar to the positive rate $(73.1 \%)$ of anti-AdHu 5 NAbs observed in 1,154 healthy adults from six regions in China (13). No significant difference in the seroprevalence rates of NAbs to AdHu5 between healthy adult volunteers and cancer patients was observed (71.57 vs. $67.05 \%$, $P>0.05)$. However, the seroprevalence rate for AdC68 NAbs was $37.27 \%$ among all the human subjects, which was much lower than that for AdHu5 NAbs. The seroprevalence rates of NAbs against AdC68 in the cancer patient samples were statistically higher than that of healthy adult volunteers (43.64 vs. $23.53 \%$, $P=0.000)$. Similar results were reported in an early study which showed that seroprevalence rate of NAbs against AdC6 and AdC7 was higher in primary hepatocellular carcinoma than that in healthy volunteers (22). Although there is no clear explanation for this phenomenon, cross-reactivity of various viral infections
TABLE 8 | Titers of the AdHu5 and AdC68 neutralizing antibody in different age groups.

\begin{tabular}{lrccc}
\hline Age groups (years) & $\mathbf{N}$ & $\begin{array}{c}\text { AdHu5 (\%) } \\
\text { medium }\end{array}$ & $\begin{array}{c}\text { AdC68 (\%) } \\
\text { medium }\end{array}$ & $P$-value \\
\hline Total samples & 36 & 20 & 0 & 0.001 \\
18-39 & 113 & 80 & 0 & 0.000 \\
$40-49$ & 218 & 80 & 10 & 0.000 \\
50-59 & 274 & 80 & 10 & 0.000 \\
$\geq 60$ & & & & \\
Healthy adult volunteers & 11 & 160 & 0 & 0.007 \\
18-39 & 27 & 160 & 0 & 0.000 \\
$40-49$ & 74 & 320 & 0 & 0.000 \\
50-59 & 92 & 160 & 0 & 0.000 \\
$\geq 60$ & & & & \\
Cancer patients & 25 & 10 & 0 & 0.026 \\
18-39 & 86 & 40 & 0 & 0.000 \\
$40-49$ & 144 & 80 & 10 & 0.000 \\
$50-59$ & 182 & 80 & 20 & 0.000 \\
$\geq 60$ & & & &
\end{tabular}

may be involved because of the lower titer distribution of NAbs to AdC68.

A total of 440 cancer subjects were enrolled in this study, which included eight types of cancer. Compared with subjects with other types of cancer, the seroprevalence rates of AdC68 NAbs were much lower in laryngeal (25.71\%), esophageal $(32.89 \%)$, and cervical $(26.67 \%)$ cancer subjects, which encourages the clinical use of AdC68-based vectors as antitumor vaccine carriers. Because few subjects with oropharyngeal, colon, and rectal cancer were enrolled in this study, the distribution difference of AdC68 NAbs among the different cancer types should be studied with much more samples.

Furthermore, we analyzed the seroprevalence of AdC68 NAbs in different clinical stages of cancer subjects. The seroprevalence rates of AdC68 NAbs were significantly higher than AdHu5 NAbs in stages I, II, and III. Although the seroprevalence rate of AdC68 NAbs was much lower than that of AdHu5 NAbs in stage-IV 
subjects, there was no significant difference between them. This may be due to the small number of subjects from stage IV.

Interestingly, the seroprevalence rates of NAbs to AdC68 in the lung squamous cell carcinoma samples were statistically higher than those in the lung adenocarcinoma samples (70.00 vs. $35.00 \%, P=0.049)$. These results indicated that the AdC68 vector is more suitable as a vaccine carrier for lung adenocarcinoma than that for lung squamous cell carcinoma.

Previous studies showed that NAbs against AdHu5 were commonly present at high titers in human sera, while the NAb titers against AdC68 were much lower (31). High-titer NAbs against adenovirus could affect the immunogenicity and reduce the effectiveness of the vaccine in vivo (32-35). Zhang et al. reported that $59.2 \%$ of Chinese subjects exhibited AdHu $5 \mathrm{NAb}$ titers $>160$, and $21.2 \%$ of the subjects exhibited titers $>1,000$. They also showed that $2.0 \%$ of Chinese subjects exhibited AdC68 NAb titers $>160$, and $0.0 \%$ exhibited titers $>1,000$ (13). In our study, $32.88 \%$ of the cancer subjects exhibited AdHu5 NAb titers $>160$, and $6.78 \%$ exhibited AdHu5 NAb titers $>1,000$. In total, $4.17 \%$ of the cancer subjects harbored AdC68 NAb titers $>160$, and $0.0 \%$ harbored AdC68 NAb titers $>1,000$. Taken together, we concluded that the AdC68 $\mathrm{NAb}$ titers from the positive samples were much lower than the AdHu 5 NAb titers.

In addition, we assessed the seroprevalence of NAbs against AdHu5 and AdC68 in different age groups. It showed similar results with the previous studies (13). The titer of NAbs against AdC68 was significantly lower than AdHu5 in the same age groups. However, there were no significant differences with the titer of NAbs against AdHu5 and AdC68 in different age groups.

In conclusion, the seroprevalence rates of NAbs against AdC68 were much lower than those against AdHu5 in cancer subjects, especially in lung adenocarcinomas, laryngeal, esophageal, and

\section{REFERENCES}

1. Wold WS, Toth K. Adenovirus vectors for gene therapy, vaccination and cancer gene therapy. Curr Gene Ther (2013) 13(6):421-33. doi:10.2174/15665 23213666131125095046

2. Xiang B, Baybutt TR, Berman-Booty L, Magee MS, Waldman SA, Alexeev VY, et al. Prime-boost immunization eliminates metastatic colorectal cancer by producing high-avidity effector CD8+ T Cells. JImmunol (2017) 198(9):3507-14. doi:10.4049/jimmunol.1502672

3. Smaill F, Jeyanathan M, Smieja M, Medina MF, Thanthrige-Don N, Zganiacz A, et al. A human type 5 adenovirus-based tuberculosis vaccine induces robust $\mathrm{T}$ cell responses in humans despite preexisting anti-adenovirus immunity. Sci Transl Med (2013) 5(205):205ra134. doi:10.1126/scitranslmed.3006843

4. Shiver JW, Fu TM, Chen L, Casimiro DR, Davies ME, Evans RK, et al. Replicationincompetent adenoviral vaccine vector elicits effective anti-immunodeficiencyvirus immunity. Nature (2002) 415(6869):331-5. doi:10.1038/415331a

5. Xiang ZQ, Yang Y, Wilson JM, Ertl HC. A replication-defective human adenovirus recombinant serves as a highly efficacious vaccine carrier. Virology (1996) 219(1):220-7. doi:10.1006/viro.1996.0239

6. Harro CD, Robertson MN, Lally MA, O'Neill LD, Edupuganti S, Goepfert PA, et al. Safety and immunogenicity of adenovirus-vectored near-consensus HIV type 1 clade B gag vaccines in healthy adults. AIDS Res Hum Retroviruses (2009) 25(1):103-14. doi:10.1089/aid.2008.0212 cervical cancers. These results could provide useful insights for developing future AdC68-based cancer vaccines. Furthermore, before the clinical trial, the biological distribution of carrier as well as acute and chronic toxic reactions should be evaluated in mice and non-human primates. The prime-boost program should also be evaluated to strength the immune effect.

\section{ETHICS STATEMENT}

This study was approved by the Ethical Committee of TMUCIH. Written informed consent was obtained from each subject in accordance with the Declaration of Helsinki.

\section{AUTHOR CONTRIBUTIONS}

$\mathrm{HZ}$ and HS designed the study protocol. HZ wrote this manuscript. HZ, CX, and XL performed the experiments. FW and $\mathrm{HZ}$ analyzed the collected data. XR revised the manuscript. All authors read and approved the final manuscript.

\section{FUNDING}

This work was supported by National Key Technology R\&D Program (No.2015BAI12B12), National Major Scientific and Technological Special Project for Significant New Drugs Development (No.2015ZX09102018), Tianjin anticancer Major Scientific Project (No.14ZCDZSY00166) and the National Natural Science Funds (No.81401888).

\section{SUPPLEMENTARY MATERIAL}

The Supplementary Material for this article can be found online at http://www.frontiersin.org/articles/10.3389/fimmu.2018.00335/ full\#supplementary-material.

7. Small JC, Haut LH, Bian A, Ertl HC. The effect of adenovirus-specific antibodies on adenoviral vector-induced, transgene product-specific $\mathrm{T}$ cell responses. J Leukoc Biol (2014) 96(5):821-31. doi:10.1189/jlb.1A0813451RR

8. Snook AE, Baybutt TR, Hyslop T, Waldman SA. Preclinical evaluation of a replication-deficient recombinant adenovirus serotype 5 vaccine expressing guanylate cyclase C and the PADRE T-helper epitope. Hum Gene Ther Methods (2016) 27(6):238-50. doi:10.1089/hgtb.2016.114

9. Appaiahgari MB, Pandey RM, Vrati S. Seroprevalence of neutralizing antibodies to adenovirus type 5 among children in India: implications for recombinant adenovirus-based vaccines. Clin Vaccine Immunol (2007) 14(8):1053-5. doi:10.1128/CVI.00173-07

10. Farina SF, Gao GP, Xiang ZQ, Rux JJ, Burnett RM, Alvira MR, et al. Replication-defective vector based on a chimpanzee adenovirus. J Virol (2001) 75(23):11603-13. doi:10.1128/JVI.75.23.11603-11613.2001

11. Xiang Z, Li Y, Gao G, Wilson JM, Ertl HC. Mucosally delivered E1-deleted adenoviral vaccine carriers induce transgene product-specific antibody responses in neonatal mice. J Immunol (2003) 171(8):4287-93. doi:10.4049/ jimmunol.171.8.4287

12. Emmer KL, Wieczorek L, Tuyishime S, Molnar S, Polonis VR, Ertl HC. Antibody responses to prime-boost vaccination with an HIV-1 gp145 envelope protein and chimpanzee adenovirus vectors expressing HIV-1 gp140. AIDS (2016) 30(16):2405-14. doi:10.1097/QAD.0000000000001224 
13. Zhang S, Huang W, Zhou X, Zhao Q, Wang Q, Jia B. Seroprevalence of neutralizing antibodies to human adenoviruses type- 5 and type- 26 and chimpanzee adenovirus type-68 in healthy Chinese adults. J Med Virol (2013) 85(6):1077-84. doi:10.1002/jmv.23546

14. Xing M, Wang X, Chi Y, Zhou D. Gene therapy for colorectal cancer using adenovirus-mediated full-length antibody, cetuximab. Oncotarget (2016) 7(19):28262-72. doi:10.18632/oncotarget.8596

15. Tatsis N, Blejer A, Lasaro MO, Hensley SE, Cun A, Tesema L, et al. A CD46binding chimpanzee adenovirus vector as a vaccine carrier. Mol Ther (2007) 15(3):608-17. doi:10.1038/sj.mt.6300078

16. Tang X, Zhang H, Song Y, Zhou D, Wang J. Hemagglutinin-targeting artificial microRNAs expressed by adenovirus protect mice from different clades of H5N1 infection. Mol Ther Nucleic Acids (2016) 19(5):e311. doi:10.1038/ mtna.2016.25

17. Zhou D, Wu TL, Emmer KL, Kurupati R, Tuyishime S, Li Y, et al. Hexonmodified recombinant E1-deleted adenovirus vectors as dual specificity vaccine carriers for influenza virus. Mol Ther (2013) 21(3):696-706. doi:10.1038/ mt.2012.248

18. Zhang C, Yang Y, Chi Y, Yin J, Yan L, Ku Z, et al. Hexon-modified recombinant E1-deleted adenoviral vectors as bivalent vaccine carriers for coxsackievirus A16 and enterovirus 71. Vaccine (2015) 33(39):5087-94. doi:10.1016/j. vaccine.2015.08.016

19. Zhou D, Cun A, Li Y, Xiang Z, Ertl HC. A chimpanzee-origin adenovirus vector expressing the rabies virus glycoprotein as an oral vaccine against inhalation infection with rabies virus. Mol Ther (2006) 14(5):662-72. doi:10.1016/j. ymthe.2006.03.027

20. Pinto AR, Fitzgerald JC, Giles-Davis W, Gao GP, Wilson JM, Ertl HC. Induction of CD8+ $\mathrm{T}$ cells to an HIV-1 antigen through a prime boost regimen with heterologous E1-deleted adenoviral vaccine carriers. J Immunol (2003) 171(12):6774-9. doi:10.4049/jimmunol.171.12.6774

21. Zhang Y, Ertl HC. The effect of adjuvanting cancer vaccines with herpes simplex virus glycoprotein D on melanoma-driven CD8+ T cell exhaustion. J Immunol (2014) 193(4):1836-46. doi:10.4049/jimmunol.1302029

22. Jian L, Zhao Q, Zhang S, Huang W, Xiong Y, Zhou X, et al. The prevalence of neutralising antibodies to chimpanzee adenovirus type 6 and type 7 in healthy adult volunteers, patients with chronic hepatitis B and patients with primary epatocellular carcinoma in China. Arch Virol (2014) 159:465-70. doi:10.1007/ s00705-013-1828-y

23. Ersching J, Hernandez MI, Cezarotto FS, Ferreira JD, Martins AB, Switzer WM, et al. Neutralizing antibodies to human and simian adenoviruses in humans and new-world monkeys. Virology (2010) 407(1):1-6. doi:10.1016/j. virol.2010.07.043

24. Xiang Z, Li Y, Cun A, Yang W, Ellenberg S, Switzer WM, et al. Chimpanzee adenovirus antibodies in humans, sub-Saharan Africa. Emerg Infect Dis (2006) 12(10):1596-9. doi:10.3201/eid1210.060078

25. Yu B, Zhou Y, Wu H, Wang Z, Zhan Y, Feng X, et al. Seroprevalence of neutralizing antibodies to human adenovirus type 5 in healthy adults in China. J Med Virol (2012) 84(9):1408-14. doi:10.1002/jmv.23325

26. Davis AR, Wivel NA, Palladino JL, Tao L, Wilson JM. Construction of adenoviral vectors. Mol Biotechnol (2001) 18(1):63-70. doi:10.1385/ MB:18:1:63

27. Abbink P, Lemckert AA, Ewald BA, Lynch DM, Denholtz M, Smits S, et al. Comparative seroprevalence and immunogenicity of six rare serotype recombinant adenovirus vaccine vectors from subgroups B and D. J Virol (2007) 81(9):4654-63. doi:10.1128/JVI.02696-06

28. Dudareva M, Andrews L, Gilbert SC, Bejon P, Marsh K, Mwacharo J, et al. Prevalence of serum neutralizing antibodies against chimpanzee adenovirus 63 and human adenovirus 5 in Kenyan children, in the context of vaccine vector efficacy. Vaccine (2009) 27(27):3501-4. doi:10.1016/j.vaccine.2009.03.080

29. Kostense S, Koudstaal W, Sprangers M, Weverling GJ, Penders G, Helmus N, et al. Adenovirus types 5 and 35 seroprevalence in AIDS risk groups supports type 35 as a vaccine vector. AIDS (2004) 18(8):1213-6. doi:10.1097/00002030200405210-00019

30. Mast TC, Kierstead L, Gupta SB, Nikas AA, Kallas EG, Novitsky V, et al. International epidemiology of human pre-existing adenovirus (Ad) type-5, type-6, type-26 and type-36 neutralizing antibodies: correlates of high Ad5 titers and implications for potential HIV vaccine trials. Vaccine (2010) 28(4):950-7. doi:10.1016/j.vaccine.2009.10.145

31. Nwanegbo E, Vardas E, Gao W, Whittle H, Sun H, Rowe D, et al. Prevalence of neutralizing antibodies to adenoviral serotypes 5 and 35 in the adult populations of The Gambia, South Africa, and the United States. Clin Diagn Lab Immunol (2004) 11(2):351-7. doi:10.1128/CDLI.11.2.351-357.2004

32. Barouch DH, McKay PF, Sumida SM, Santra S, Jackson SS, Gorgone DA, et al. Plasmid chemokines and colony-stimulating factors enhance the immunogenicity of DNA priming-viral vector boosting human immunodeficiency virus type 1 vaccines. J Virol (2003) 77(16):8729-35. doi:10.1128/ JVI.77.16.8729-8735

33. Casimiro DR, Chen L, Fu TM, Evans RK, Caulfield MJ, Davies ME, et al. Comparative immunogenicity in rhesus monkeys of DNA plasmid, recombinant vaccinia virus, and replication-defective adenovirus vectors expressing a human immunodeficiency virus type 1 gag gene. J Virol (2003) 77(11):6305-13. doi:10.1128/JVI.77.13.7663-7668.2003

34. McElrath MJ, De Rosa SC, Moodie Z, Dubey S, Kierstead L, Janes H, et al. HIV-1 vaccine-induced immunity in the test-of-concept step study: a case-cohort analysis. Lancet (2008) 372(9653):1894-905. doi:10.1016/ S0140-6736(08)61592-5

35. Quirk EK, Mogg R, Brown DD, Lally MA, Mehrotra DV, DiNubile MJ, et al. HIV seroconversion without infection after receipt of adenovirus-vectored HIV type 1 vaccine. Clin Infect Dis (2008) 47(12):1593-9. doi:10.1086/593313

Conflict of Interest Statement: Tianjin Bioroc Pharmaceutical \& Biotech Company is mainly engaged in research and development of cancer immunotherapy and related products. Tianjin Genstar Vaccine Limited Liability Company is mainly engaged in research and development of viral vector-based therapeutic tumor vaccines. Tianjin Genstar Vaccine Limited Liability Company is the subsidiary holding by Tianjin Bioroc Pharmaceutical \& Biotech Company. CX, XL, NW, and HS are employees of Tianjin Genstar Vaccine Limited Liability Company. HZ, FW, and XR are scientific researchers of Tianjin Medical University Cancer Institute \& Hospital and declare that they have no competing interests.

Copyright (c) 2018 Zhao, Xu, Luo, Wei, Wang, Shi and Ren. This is an open-access article distributed under the terms of the Creative Commons Attribution License (CC BY). The use, distribution or reproduction in other forums is permitted, provided the original author(s) and the copyright owner are credited and that the original publication in this journal is cited, in accordance with accepted academic practice. No use, distribution or reproduction is permitted which does not comply with these terms. 